\section{OP0304-PARE DEVELOPMENT OF INTERACTIVE DECISION AID TOOL FOR MOTHERHOOD AND PARENTHOOD AMONG PATIENTS LIVING WITH AUTOIMMUNE RHEUMATIC DISEASES}

\begin{abstract}
Y. El-Miedany ${ }^{1,2}$, M. El Gaafary ${ }^{3}$, N. El Aroussy ${ }^{4}$, S. Bahlas ${ }^{5}$, D. Palmer ${ }^{6}$. ${ }^{1}$ Canterbury Christ Church University, Rheumatology, Canterbury, United Kingdom; ${ }^{2}$ King's College London, Education, London, United Kingdom; ${ }^{3}$ Faculty of Medicine, Ain Shams University, Community and Public health, Cairo, Egypt; ${ }^{4}$ Faculty of Medicine, Ain Shams University, Rheumatology and Rehabilitation, Cairo, Egypt; ${ }^{5}$ King Abdulaziz University, Rheumatology, Jeddah, Saudi Arabia; ${ }^{6}$ North Middlesex University Hospital, Rheumatology, London, United Kingdom
\end{abstract}

Background: There are clear educational pregnancy-related needs for both women and men living with Autoimmune Rheumatic Diseases (ARDs) who seek specific advice from their health care providers. Most of the time those subjects would seek specific information regarding their chances of pregnancy, impact of the disease, as well as safety of their medications during pregnancy and breast feeding. They also require emotional and physical support as well as advice towards practical coping strategies to manage pain and disease flares particularly during the postnatal period.

Objectives: To evaluate the effectiveness of a new interactive pregnancy decision aid (DA) developed for both men and women living with inflammatory arthritis. Methods: Driven by the Cochrane review of patient decision aids (DA) and the International Patient Decision Aids framework (IPDAS), which identify shared decision making (SDM) instrument as evidence-based tools designed to help people engage in deliberative management-related decision making by providing information on the options and outcomes relevant to health status. This project involved an overall three-phase SDM aid development. It has been set up for both men and women and included information on pregnancy, contraception and breast feeding. The first phase included the development of the decision tool and review by expert faculty composed of: decision experts, patient representatives, policy makers, and patients living with RA, PsA, AS and SLE. The second phase was a pilot testing of the tool in both online and paper format styles. The third phase involved an evaluation of the decision support tool and its impact on the patients. The DA adopted an interactive style with embedded video recordings (this was converted into text in the paper format). All the patient completed these questionnaires: the ReproKnow, Decisional Conflict Scale (DCS), Hospital Anxiety and Depression Scale (HADS), and Arthritis Self-Efficacy Scale (ASES) pre- and post-intervention. A control group of 92 patients (19 men and 71 women) managed according to the standard protocols was also included. Comprehensibility of the video recordings were also assessed using VAS (0-10). Results: 126 patients shared in this work (101 women and 25 men). The patients who received the DA had an $81 \%$ (women) and $83 \%$ (men) increase in ReproKnow scores and a $76 \%$ decrease in scores on the decisional conflict (DCS), compared to the control group ( $10 \%$ and $11 \%$ respectively); $p<0.001$. There were no changes in the scores of depression and anxiety symptoms $(p=0.873)$ indicating no adverse psychological impact detected. There was no significant difference in terms of self-efficacy assessment $p=0.481$. The patients rated the comprehensibility of the video recording as high $(9.4 / 10)$

Conclusion: The DA which was developed to support pregnancy and breast feeding decision-making for patients with ARDs. The interactive SDM aid was found to be a simple, user-friendly tool which can be implemented in standard clinical practice. The interactive style made it more comprehensible to the patients. It is consistent with the IPDAS criteria. Results revealed its effectiveness in improving relevant knowledge and reducing decisional conflict without causing distress to the patients.

Disclosure of Interests: None declared

DOI: 10.1136/annrheumdis-2021-eular.513

\section{Controversies on phenotypes in PsA}

\begin{tabular}{|l|l|}
\hline OP0305 & VALIDATION OF THE PSORIASIS EPIDEMIOLOGY \\
SCREENING TOOL (PEST) AND THE NEW EARLY \\
ARTHRITIS FOR PSORIATIC PATIENTS (EARP) IN \\
PEDIATRIC POPULATION - PILOT STUDY
\end{tabular}

Y. Butbul' ${ }^{1}{ }^{1}$ The Ruth and Bruce Rappaport Faculty of Medicine, Technion Israel Institute of Technology; PEDIATRIC RHEUMATOLOGY UNIT, Haifa, Israel

Background: Juvenile Psoriatic Arthritis (JPsA) is an inflammatory arthritis associated with irreversible joint damage among the pediatric population, which is associated with psoriasis in most cases.

While there are few validated screening tools for diagnosis of arthritis for adult patients with psoriasis, those screening tools were never evaluated in children.
Objectives: The aims of this study were to evaluate two screening tools among pediatric patients with psoriasis.

Methods: Thirty-nine patients with the diagnosis of psoriasis were administered two screening questionnaires: the new Early Arthritis for Psoriatic Patients (EARP) questionnaire and the Psoriatic Arthritis Screening and Evaluation (PASE) questionnaire.

All patients were evaluated by a rheumatologist for the diagnosis of JPsA, and the accuracy of the two questionnaires compared.

Results: The 4/39 (10.1\%) patients diagnosed with JPsA had a PEST questionnaire score of $\geq 3$, compared to a median PEST score of the patients without the diagnosis of JPsA of $0(0-2)$. Thus both the sensitivity and specificity of the PEST in diagnosing JPsA were $100 \%$.

For the EARP questionnaire, 8/39 patients had a screening questionnaire score of $\geq 3$, suggestive of JPsA, four were true positive, and four false positive. Thus, the sensitivity and specificity of EARP in diagnosing JPsA were $100 \%$ and $89 \%$, respectively.

Conclusion: Both the PEST and EARP questionnaires were easy to use and had high sensitivity for pediatric population with psoriasis, however the PEST questionnaire had a higher specificity than the EARP.

Disclosure of Interests: None declared.

DOI: 10.1136/annrheumdis-2021-eular.4165

\section{OP0306 \\ ETANERCEPT AND ADALIMUMAB IN THE TREATMENT OF JUVENILE PSORIATIC ARTHRITIS}

K. Aleksanyan ${ }^{1}$, S. Chebysheva ${ }^{1}$, O. Sukhovyova ${ }^{1}$, E. Zholobova ${ }^{1} .{ }^{1}$ I.M. Sechenov First Moscow State Medical University, Clinical Institute of Child Health named after N.F. Filatov, Moscow, Russian Federation

Background: Juvenile psoriatic arthritis (JPsA) accounts for 4 to $9 \%$ of children with juvenile arthritis (1). Biologics, particularly TNF- $\alpha$ inhibitors, have proved to be effective and safe for treatment of psoriatic arthritis in adults (2). However, no comprehensive evidence exists on the comparative effectiveness and safety of TNF- $\alpha$ inhibitors in the treatment of JPsA, which challenges a decision of what biologic should be prescribed to children with JPsA.

Objectives: To study the comparative effectiveness of etanercept and adalimumab in patients with JPsA with respect to both the articular and skin manifestations.

Methods: Our observational (2012-2019), parallel-group, open-label, single-centre cohort clinical study involved 34 patients $(2-13$ y/o) with JPsA who met Vancouver and I/E criteria and received etanercept SC $(0.8 \mathrm{mg} / \mathrm{kg} \mathrm{QW}$, max $50 \mathrm{mg}$ per week) or adalimumab SC ( $\max 40 \mathrm{mg}$ Q2W), both in combination with methotrexate $\left(10-15 \mathrm{mg} / \mathrm{m}^{2} \mathrm{QW}\right)$. Intention-to-treat analysis was used to evaluate treatment efficacy at months 6,12 , and 18 after biologic therapy initiation. To assess articular manifestations of JPsA, we applied ACRpedi criteria; to estimate the surface area of involved skin and severity of psoriasis - the PASI score.

Results: We observed 34 patients with JPsA ( 2 to $13 \mathrm{y} / 0$ ), who were initiated by biological therapy (etanercept $[n=18-$ group 1] and adalimumab $[n=16-$ group 2]) (Table 1, Figure 1).

Table 1. Clinical and demographic characteristics of patients with JPsA before biologic therapy initiation $(n=34)$

\begin{tabular}{lll}
\hline clinical and demographic indicators & Group 1 & Group 2 \\
& (Etanercept) & (Adalimumab) \\
& M $\pm \delta /$ Me (Q1-Q3) & M $\pm \delta /$ Me (Q1-Q3) \\
& $12: 6(2: 1)$ & $10: 6(1,7: 1)$ \\
\hline Girl/Boy Ratio & $7,58 \pm 3,7$ & $5,8 \pm 3$ \\
Average age, years & $3,0(1,4-6,6)$ & $3,5 \pm 2$ \\
Duration of the disease, years & 0 & $5(31,3 \%)$ \\
Uveitis (n, \%) & $2(11 \%)$ & $8(50 \%)$ \\
ANA positivity (n, \%) & 0 & 0 \\
RF positivity (\%) & $8,0(5-16,5)$ & $5,5(3-8,5)$ \\
No. active joints & $9(5,75-18,25)$ & $6(3,5-10,5)$ \\
No. joints with LOM & $70 \pm 15$ & $65,9 \pm 12,7$ \\
PGA of disease activity, mm & $71,5(65-90)$ & $72,5 \pm 14$ \\
Parent's global assessment of the child's pain, VAS & $1,34(1,1-1,68)$ & $1,25(0,93-1,44)$ \\
CHAQ & $7,0(4-13)$ & $8(3-13)$ \\
Psoriasis BSA, \% & $5,7(3-8,2)$ & $9(5-19)$ \\
PASI score & $28(20,75-40)$ & $24,5(21-32)$ \\
ESR, mm/h &
\end{tabular}

ANA - antinuclear antibodies, RF - rheumatoid factor, LOM - limitation of motion, PGA - physician's global assessment; VAS - visual analog scale, CHAQ - Childhood Health Assessment Questionnaire; BSA - body surface area; PASI - Psoriasis Area Severity Index, ESR - erythrocyte sedimentation rate; CRP - C-reactive protein. 